\title{
A new method for determining mass-to-light ratios of nearly face-on spiral galaxies
}

\author{
$\mathrm{TaOHu}^{1}$, Qiu-He Peng ${ }^{1,2,3}$, and Ying-He Zhao ${ }^{1}$ \\ 1 Department of Astronomy, Nanjing University, Nanjing 210093, PR China \\ e-mail: taohu@nju.edu.cn \\ 2 Joint Astrophysics Center of Chinese Academy of Science-Peking University, Beijing 100871, PR China \\ 3 The Open Laboratory of Cosmic Ray and High Energy Astrophysics, Chinese Academy of Science
}

Received 12 August 2005 / Accepted 8 December 2005

\section{ABSTRACT}

Aims. This letter gives a new method for determining mass-to-light ratios of nearly face-on spiral galaxies.

Methods. The method is based on the effective thickness of the galactic disk, the distribution of the vertical velocity dispersion, and the surface brightness of a spiral galaxy.

Results. As examples, the results of the determination of NGC 1566 and NGC 5247 in $B$-band are presented, and their mass-to-light ratios are $4.86 \sim 8.99 M_{\odot} L_{\odot}^{-1}$ and $5.02 \sim 6.90 M_{\odot} L_{\odot}^{-1}$ respective.

Key words. galaxy: disk - galaxies: fundamental parameters - galaxies: spiral - galaxies: structure galaxies: individual: NGC 1566, NGC 5247

\section{Introduction}

Van der Kruit \& Searle (1981a,b) investigated surface photometry of edge-on spiral galaxies, they fitted the $z$ distribution of light at each $r$ of the disk by a model of a locally isothermal sheet. Let $\Phi_{z}$ denote the gravitational force in the $z$-direction as a function of $z, \rho$ the space density of matter and $\left\langle V_{z}^{2}\right\rangle^{1 / 2}$ the dispersion in the velocities in the $z$-direction. From the case Poisson's and Liouville's equations, it could be reduced to

$\frac{\partial \Phi_{z}}{\partial z}=-4 \pi G \rho(z)$

$\frac{\partial \rho}{\partial z}=\frac{\rho V_{z}}{\left\langle V_{z}^{2}\right\rangle}$,

with $\left\langle V_{z}^{2}\right\rangle^{1 / 2}$ independent of $z$-direction, and the solution is (Camm 1950; Spitzer 1942)

$\rho(r, z)=\rho(r, 0) \operatorname{sech}^{2}\left(\frac{z}{z_{0}}\right)$

with

$z_{0}=\frac{\left\langle V_{z}^{2}\right\rangle^{1 / 2}}{[2 \pi G \rho(r, 0)]^{1 / 2}}$,

(4) $\gamma=\frac{0.462\left\langle V_{z}^{2}\right\rangle}{\pi G z_{0} I_{0} \mathrm{e}^{-r / r_{\mathrm{d}}}}$

$z_{0}$ is effective thickness of a galactic disk $\left(H=z_{0} / 2\right.$ is scale height of a galactic disk). disk

By integrating Eq. (3), we have the total surface density of

$\sigma(r)=\frac{0.462 \times \sqrt{2} \rho(r, 0)^{1 / 2}\left\langle V_{z}^{2}\right\rangle^{1 / 2}}{(\pi G)^{1 / 2}}$,

so Eq. (4) can be reduced to

$z_{0}=\frac{0.462\left\langle V_{z}^{2}\right\rangle}{\pi G \sigma(r)}$

Van der Kruit \& Freeman (1986) pointed out that the mass-tolight ratio $(\gamma=M / L)$ of old disk is approximately constant, in good approximation independent of position along the radius. When a spiral galaxy is nearly face-on, the total surface density can be taken as

$\sigma(r)=\gamma I(r)=\gamma I_{0} \mathrm{e}^{-r / r_{\mathrm{d}}}$,

with $I(r)$ the surface brightness and $r_{\mathrm{d}}$ the scale length of galactic disk. Therefore, from Eqs. (6) and (7), we can find

if we obtain the parameters $z_{0}, V_{z}, I_{0}$, and $r_{\mathrm{d}}$, mass-to-light ratio $\gamma$ could be calculated by Eq. (8). 


\section{Model and data reduction}

\subsection{The effective thickness $z_{0}$}

Van der Kruit \& Searle (1981a) proposed a method to determine the scale heights of edge-on disk galaxies. It is based on measuring surface brightness that is distributed with exponential function of radius. A method for determining the scale heights of spiral galaxies observed non-edge-on was proposed by Peng (1988) on the basis of the asymptotic expression of the perturbed gravitational potential. Zhao et al. (2004) re-investigate the method based on the rigorous expression of the perturbed gravitational potential. We have already obtained perturbed gravitational potential for such a logarithmic density disturbance via the Poisson's equation for the galactic disk with finite thickness (Peng et al. 1978, 1979), the perturbed gravitational potential may be expressed

$$
\begin{aligned}
V_{\alpha}(r, \phi, z=0, t)= & -2 \pi G A \exp [\mathrm{i}(\omega t-m \phi+\Lambda \ln r)] . \\
& \operatorname{Re}[g(\Lambda, m ; \alpha r)],
\end{aligned}
$$

where

$$
\begin{aligned}
g(\Lambda, m ; \alpha r)= & \exp (\mathrm{i} \Lambda \ln 2) \frac{\Gamma\left(\frac{1+m+\mathrm{i} \Lambda}{2}\right)}{\Gamma\left(\frac{1+m-\mathrm{i} \Lambda}{2}\right)} . \\
& \int_{0}^{\infty} J_{m}(x) \frac{\exp (-\mathrm{i} \Lambda \ln x)}{x\left(1+\frac{x}{\alpha r}\right)} \mathrm{d} x,
\end{aligned}
$$

here, $\alpha$ is thickness factor of a spiral galactic disk, $\Gamma(x)$ and $J_{m}(x)$ are the usual Gamma and Bessel functions, respective. For an infinitely thin disk $(\alpha \rightarrow+\infty)$, the Eq. (9) has a simplified form as same as the expression given by Kalnajs (1971)

$$
\begin{aligned}
V_{\alpha \rightarrow+\infty}(r, \phi, z=0, t)= & -2 \pi G A \exp [\mathrm{i}(\omega t-m \phi \\
& +\Lambda \ln r)] \frac{1}{\sqrt{m^{2}+\Lambda^{2}}} .
\end{aligned}
$$

The ratio $(\eta)$ of the amplitude for the perturbed gravitational potential for a disk galaxy with finite thickness to that of an infinitely thin disk at the forbidden radius $r_{0}$ is (Zhao et al. 2004)

$\eta=\frac{V_{\alpha}\left(\alpha, m, \Lambda, r_{0}\right)}{V_{\alpha \rightarrow \infty}\left(m, \Lambda, r_{0}\right)}=\operatorname{Re}\left[g\left(\Lambda, m ; \alpha r_{0}\right)\right] \sqrt{m^{2}+\Lambda^{2}}$,

it can be obtained the effective thickness $\left(z_{0}=2 / \alpha\right)$ through Eq. (12) by determining the winding parameter $(\Lambda)$, the number of spiral arms $(m)$, and the forbidden radius (A spiral arm can not extend to the forbidden region with radius $r_{0}$ to the galactic center.). $\eta$ was assumed to be 0.5 based on the comparison between $z_{0}$ and $r_{0}$ which have been observed and measured for Milky Way and Andromeda Nebula (M31). In fact, we use the same values of the parameters of 71 spiral galaxies given by Ma et al. (1998) to calculate the factor $\eta$ by Eq. (12), and obtain the average $\eta \approx 0.486$. In this paper, we take the average value of 0.5 and 0.486 , i.e., $\bar{\eta}=0.493$.

It is well known that spiral arms can be represented by equiangular spirals on the galactic disk. In Fig. 1, we determine the inclinations and the winding parameters of the spiral galaxies NGC 1566 and NGC 5247 ( $B$-band) by fitting the curves of spiral arms in their images, i.e., it is the optimum inclination of a spiral galaxy, which is corresponding to the best fitted spiral curves.
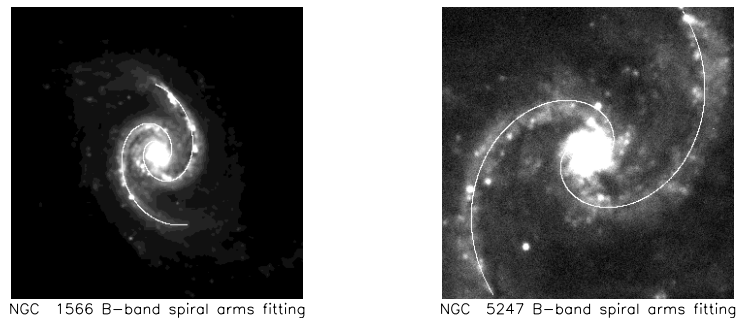

Fig. 1. Images of NGC 1566 and NGC 5247 in B-band with superposed fitted curves of spiral arms.

Table 1. $V_{z}$ and parameters of surface brightness of NGC 1566 and NGC 5247 ( $B$-band).

\begin{tabular}{lcccccc}
\hline \hline NGC & Band & $\begin{array}{c}d \\
(\mathrm{Mpc})\end{array}$ & $\begin{array}{c}r \\
(\mathrm{kpc})\end{array}$ & $\begin{array}{c}V_{z, r} \\
\left(\mathrm{~km} \mathrm{~s}^{-1}\right)\end{array}$ & $\begin{array}{c}I_{0} \\
\left(L_{\odot} \mathrm{pc}^{-2}\right)\end{array}$ & $\begin{array}{c}r_{\mathrm{d}} \\
(\mathrm{kpc})\end{array}$ \\
$(1)$ & $(2)$ & $(3)$ & $(4)$ & $(5)$ & $(6)$ & $(7)$ \\
\hline 1566 & $B$ & 17.4 & $2.5 \sim 3.1$ & $60 \sim 40$ & 221 & 3.0 \\
5247 & $B$ & 16.7 & $2.4 \sim 3.2$ & $40 \sim 30$ & 225 & 3.1 \\
\hline
\end{tabular}

\section{2. $V_{z}$ and surface brightness of NGC 1566 and NGC 5247}

NGC 1566 and NGC 5247 were investigated based on studying the surface photometric and spectroscopic by Bottema (1992) and Van der Kruit \& Freeman (1986), respectively. The parameters $V_{z}, I_{0}$, and $r_{\mathrm{d}}$ we need are listed in Table 1 in this paper. As shown in Table 1 Col. 5 , the vertical velocity dispersion $V_{z}$ of NGC 1566 was obtained from Table 4 Col. 4 in Bottema's paper (1992) and NGC 5247 from Table 1 Col. 4 in Van der Kruit \& Freeman (1986), it only needs to get $V_{z, r}$ at the radius $r$ in the galactic disk. However, we have obtained the effective thickness $z_{0}$, the vertical velocity dispersion $V_{z, r}$, and the surface brightness $I(r)$ at radius $r$, the only unknown parameter is mass-to-light ratio $\gamma$ in Eq. (8).

\section{Results}

On the basis of the method proposed in this paper, we obtain the effective thicknesses $\left(z_{0}\right)$ of NGC 1566 and NGC 5247 by Eq. (12) and their mass-to-light ratios $(\gamma)$ by Eq. (8). The main results are listed in Table 2, $\gamma$ listed in Col. 8 and $z_{0}$ in Col. 7.

\section{Discussion}

1. As shown in Fig. 2 with four panels (Figs. 2a-d), Fig. 2a is displayed the radial dependence of $V_{V}=V_{\alpha} / V_{\alpha \rightarrow \infty}$ by Eqs. (9) and (11), it is noted that $V_{V}$ is the ratio for the amplitude of the gravitational potential perturbation of a disk with finite thickness to that of an infinitely thin disk. As an example, we choose a sample with two spiral arms and winding parameter $\Lambda=12.0$. The curves from the top to bottom denote the galactic disk with different effective thicknesses correspond to $z_{01}=0.20 \mathrm{kpc}$ $\left(\alpha_{01}=10.00 \mathrm{kpc}^{-1}\right), z_{02}=0.50 \mathrm{kpc}\left(\alpha_{02}=4.00 \mathrm{kpc}^{-1}\right)$, and $z_{03}=1.00 \mathrm{kpc}\left(\alpha_{03}=2.00 \mathrm{kpc}^{-1}\right)$, respectively. In 
Table 2. The effective thicknesses and the mass-to-light ratios of NGC 1566 and NGC 5247 (B-band).

\begin{tabular}{lccccccc}
\hline \hline NGC & $m$ & $\phi$ & $r_{0}$ & $\Lambda$ & $\alpha$ & $z_{0}$ & $\gamma$ \\
& & $\begin{array}{c}\left({ }^{0}\right) \\
(\mathrm{kpc})\end{array}$ & & $\begin{array}{c}\gamma \\
\left(\mathrm{kpc}^{-1}\right)\end{array}$ & $\begin{array}{c}\mathrm{kpc}) \\
\left(M_{\odot} L_{\odot}^{-1}\right)\end{array}$ \\
$(1)$ & $(2)$ & $(3)$ & $(4)$ & $(5)$ & $(6)$ & $(7)$ & $(8)$ \\
\hline 1566 & 2 & 32.3 & 0.996 & 6.82 & 7.018 & 0.285 & $4.86 \sim 8.99$ \\
5247 & 2 & 23.0 & 0.358 & 4.25 & 13.072 & 0.153 & $5.02 \sim 6.90$ \\
\hline
\end{tabular}

* Column 2: $m$ is the number of spiral arms.

* Column 3: $\phi$ is the optimum inclination of the galactic disk.

$\star$ Column 4: $r_{0}$ is the forbidden radius.

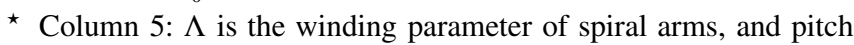
angle $\mu=\arctan (m / \Lambda)$.

* Column 6: $\alpha$ is the thickness factor, $\alpha=2 / z_{0}$.

* Column 7: $z_{0}$ is the effective thickness of the galactic disk, and here, errors $\left(\Delta z_{0}\right)$ of $z_{0}$ are $\Delta z_{0(\mathrm{NGC} 1566)}=20.3 \%$ and $\Delta z_{0(\mathrm{NGC} 5247)}=18.1 \%$.

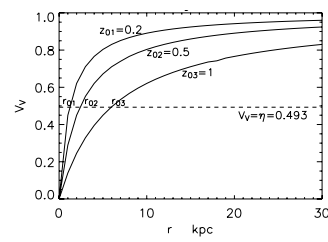

(a)

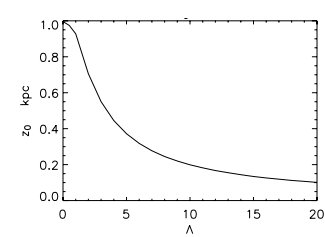

(c)

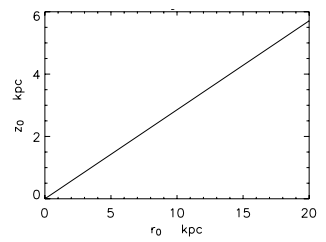

(b)

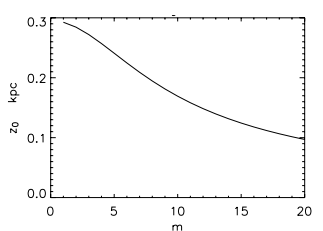

(d)
Fig. 2. Panel a) shows $V_{V}=V_{\alpha} / V_{\alpha \rightarrow \infty}$ with different $z_{0}\left(z_{0}=2 / \alpha\right)$. $V_{V}$ is the ratio for the amplitude of the gravitational potential perturbation of a disk with finite thickness to that of an infinitely thin disk. The curves from top down correspond to $z_{01}=0.20 \mathrm{kpc}$ $\left(\alpha_{01}=10.00 \mathrm{kpc}^{-1}\right), z_{02}=0.50 \mathrm{kpc}\left(\alpha_{02}=4.00 \mathrm{kpc}^{-1}\right)$, and $z_{03}=1.00 \mathrm{kpc}\left(\alpha_{03}=2.00 \mathrm{kpc}^{-1}\right)$, respectively. Panels b)-d) are three panels for NGC 1566 to illustrate the changes of $z_{0}$ with $r_{0}, \Lambda$, and $m$, respectively.

Fig. 2a, it is shown that the amplitude of gravitational perturbation for a disk with finite thickness is weaker than that of an infinitely thin disk, and the thicker the galactic disk is, the weaker the amplitude of the gravitational potential perturbation becomes. When it is close to the center of the disk $(r \rightarrow 0)$, the decrease of the amplitude of gravitational potential perturbation becomes even faster, the amplitude is too weak to stir up the self-consistent density waves due to the physical concept of density waves suggested by Lindblad, spiral arms would disappear in the forbidden region with radius $r_{0}$ to the galactic center. When $V_{V}=\eta=0.493$ as shown in Fig. 2a, the thicker the galactic disk is, the larger the forbidden radius becomes $\left(r_{03}>r_{02}>r_{01}\right)$.
2. Figures $2 b-d$ are three panels for NGC 1566. $z_{0}$ is obtained by calculating Eq. (12). Figure $2 \mathrm{~b}$ shows the effective thickness $z_{0}$ changes with $r_{0}$, keeping the other two parameters $m$ and $\Lambda$ constant at their adopted values in Table 2 . We could note that $z_{0}$ becomes thicker as the the forbidden radius $r_{0}$ is increased, it is in agreement with the conclusion obtained from Fig. 2a (when $V_{V}=\eta=0.493$, the thicker the galactic disk is, the larger the forbidden radius $r_{0}$ becomes.).

Figure $2 \mathrm{c}$ is shown the change of $z_{0}$ with $\Lambda$, keeping $m$ and $r_{0}$ constant. We can learn from Peng (1988) and Ma et al. (1998) that $z_{0} \propto r_{0} \Lambda^{-1}$, for the spiral galaxies with the same or similar value of $z_{0}$, the forbidden radii $r_{0}$ become larger as $\Lambda$ be increased, it shows some correlations with Hubble sequence. As displayed in Fig. 2c, $r_{0}$ is kept constant at the value in Table $2, z_{0}$ becomes thinner as $\Lambda$ becomes larger, it is in agreement with the method for determining the thickness of a spiral galactic disk by Peng (1988) and Ma et al. (1998).

Figure $2 \mathrm{~d}$ shows the dependence of $z_{0}$ on the parameter $m$, keeping $r_{0}$ and $\Lambda$ constant. It is noted that the larger the number of spiral arms $(m)$ becomes, the narrower the gap of the spiral arms is. It is similar to the result of $\Lambda$ increased, when $\Lambda$ is increased, the gap of the spiral arms becomes smaller. As shown in Fig. $2 \mathrm{~d}, z_{0}$ becomes thinner as $m$ becomes larger.

3. The mass-to-light ratios of NGC 1566 and NGC 5247 in $B$-band given by Bottema (1992) and Van der Kruit \& Freeman (1986) respectively are both $5.0 \sim 10.0 M_{\odot} L_{\odot}^{-1}$. As shown in Table 2 Col. 8 in this paper, we obtain the mass-to-light ratios of NGC 1566 and NGC 5247 are $4.86 \sim 8.99 M_{\odot} L_{\odot}^{-1}$ and $5.02 \sim 6.90 M_{\odot} L_{\odot}^{-1}$, respective.

4. Van de Kruit \& Searle (1981a) and de Grijs \& Van de Kruit (1996) found that, for scale heights of edge-on spirals, $H=z_{0} / 2$ is in good approximation independent of position along the major axis, but Kent et al. (1991), de Grijs \& Peletier (1997) noted that the scale height seems to increase with radius along the major axis, it seems to occur more often in early type galaxies than in later type. Shaw $\&$ Gilmore (1990) found that the radial variation of scale heights is within $\pm 3 \%$, with no dependence on color or model type. Here, we presume scale height to be approximately constant along the axis of the galactic disk, and for Eq. (8), it is obvious that mass-to-light ratio is approximately constant too, in agreement with Van der Kruit \& Freeman (1986).

Acknowledgements. We are very grateful to the referee for the careful and useful reviews of this paper, the insightful comments and suggestions have improved this paper very much. The images of NGC 1566 and NGC 5247 in B-band are both got from the NASA/IPAC Extragalactic Database (NED), which is operated by the Jet Propulsion Laboratory, California Institute of Technology, under contract with NASA. The image of NGC 1566 is obtained with the $2.5 \mathrm{~m}$ telescope at LCO, with the image size $10.2 \times 10.2 \mathrm{arcmin}$, and the image of NGC 5247 is got with the $0.9 \mathrm{~m}$ telescope at CTIO, with the image size $6.8 \times 6.8$ arcmin. This research is supported by Chinese National Science Foundation No. 10573011, No.10273006, 
and the Doctoral Program Foundation of State Education Commission of China.

\section{References}

Bottema, R. 1992, A\&A, 257, 69

Camm, G. L. 1950, MNRAS, 110, 305

de Grijs, R., \& Van der Kruit, P. C. 1996, A\&AS, 117, 19

de Grijs, R., \& Peletier, R. F. 1997, A\&A, 320, L21

Kalnajs, A. J. 1971, ApJ, 166, 275

Kent, S. M., \& Dame, T. M. 1991, ApJ, 378, 131

Ma, J., Peng, Q. H., \& Gu, Q. S. 1998, A\&AS, 130, 449
Peng, Q. H. 1988, A\&A, 206, 18

Peng, Q. H., Huang, K. L., Huang, J. H., Li, X. G., \& Su, H. J. 1978, Acta Astron. Sinica, 19, 182

Peng, Q. H., Li, X. G., Su, H. J., Huang, K. L., \& Huang, J. H. 1979, Sci. Sinica, XXII, 925

Shaw, M. A., \& Gilmore, G. 1990, MNRAS, 242, 59

Spitzer, L. 1942, ApJ, 95, 329

Van der Kruit, P. C., \& Searle, L. 1981a, A\&A, 95, 105

Van der Kruit, P. C., \& Searle, L. 1981b, A\&A, 95, 116

Van der Kruit, P. C., \& Freeman, K. C. 1986, ApJ, 303, 556

Zhao, Y. H., Peng, Q. H., \& Wang, L. 2004, Chjaa, 1, 51 